\title{
Contamination éventuelle des fromages par les mycotoxines : une revue
}

\author{
par
}

A. TANTAOUI-ELARAKI et N. KHABBAZI

\section{R és u m é}

Le lait peut être contaminé par des mycotoxines variées provenant des aliments du bétail pollués. Le cas de l'aflatoxine $\mathbf{M}_{1}$, qui a été le plus étudié, montre que ni les traitements préalables du lait (réfrigération, pasteurisation, écrémage) ni le caillage, ni le travail du caillé, ni l'affinage n'aboutissent à une élimination totale de la toxine initialement présente dans le lait.

D'autre part, les moisissures spécifiques des fromages ou de contamination, peuvent être toxinogènes. Leur développement et la synthèse de leurs toxines dépendent des conditions nutritionnelles offertes par le fromage, des conditions physiques $(\mathrm{pH}$, humidité, température) et de la compétition avec d'autres micro-organismes normaux du produit, de même que de l'utilisation éventuelle de substances antifongiques, et des techniques d'emballage et d'entreposage.

Certains travaux faisant mention de la présence de mycotoxines dans les fromages du commerce sont rapportés, et le travail est conclu par quelques réflexions sur la portée réelle du problème des mycotoxines dans les fromages, et les mesures à prendre pour limiter les risques.

Mots clés :

\section{S u m m a r y}

Possible CONTAMINATION OF CHEESE BY MYCOTOXINS

A REVIEW

Milk can contain various mycotoxins when lactating animals ingest contaminated feeds. Aflatoxin $M_{1}$ has been much more studied

Section de Technologie et d'Economie Alimentaires de Nutrition Humaine, Institut Agronomique et Vétérinaire Hassan II, B.P. 6202 Rabat - Instituts. 
than other mycotoxins. Milk treatments before processing (refrigeration, pasteurization, skimming), preparing, washing, salting and heating the curd, as well as ripening, do not completely eliminate aflatoxin $M_{I}$ initially present in raw milk.

On the other hand, either specific or abnormal molds growing on cheese can be toxigenic. Their growth and toxigenesis are related to nutritional factors (cheese components), physical conditions ( $\mathrm{pH}$, temperature, activity of water), competitive effects due to other microorganisms in the cheese, as well as use of antifungal substances and packaging and storage procedures.

The latter part of this paper summarizes some works which report occurrence of mycotoxins in commercial cheese, but the authors discuss the real extent of mycotoxin risk in this kind of products.

\section{INTRODUCTION}

Depuis que l'on sait que les femelles laitières ayant absorbé des aliments contaminés par l'aflatoxine excrètent un lait lui-même pollué, de nombreuses recherches ont été menées pour connaître le devenir de cette toxine au cours des différentes phases de la fabrication et de l'affinage des fromages.

D'autre part, la découverte de l'aflatoxine, au début des années 60 , a focalisé l'intérêt des chercheurs sur les moisissures qui se développent dans les fromages soit normalement (mycoflore spécifique) soit accidentellement (mycoflore de contamination). Le fait que certains de ces champignons se sont révélés toxinogènes n'a fait qu'accroître l'étendue et l'intensité de ces recherches.

Notre propos est de passer en revue un certain nombre de travaux relatifs à ce sujet, et d'essayer de dégager la portée réelle du problème pour le consommateur car tel est l'objectif principal de tout hygiéniste.

\section{CONTAMINATION INDIRECTE DES FROMAGES PAR LES MYCOTOXINES}

\subsection{Contamination du lait par les mycotoxines}

De nombreuses mycotoxines peuvent contaminer les aliments du bétail et se retrouver dans le lait après ingestion, suivie ou non d'une métabolisation plus ou moins prononcée.

C'est le cas, en particulier, des aflatoxines $B_{1}, B_{2}, G_{1}, G_{2}, B_{2^{a}}$ et $G_{2^{a}}$ qui sont éliminées dans le lait sous leurs formes 4-hydroxylées, respec- 
tivement $\mathrm{M}_{1}, \mathrm{M}_{2}, \mathrm{GM}_{1}, \mathrm{GM}_{2}, \mathrm{M}_{2^{\mathrm{a}}}$ et $\mathrm{GM}_{2^{\mathrm{a}}}$ (Heathcote et Hibbert, 1978) ; l'aflatoxicol peut également être éliminé suite à l'ingestion d'aflatoxine $\mathrm{B}_{1}\left(\mathrm{AFB} \mathrm{B}_{1}\right)$.

C'est également le cas de l'ochratoxine A rejetée dans le lait telle quelle et sous forme d'ochratoxine $\alpha$ (Ribelin, 1978), de la zéaralénone qui se retrouve dans la sécrétion lactée inchangée et sous forme de $\alpha$ et $\beta$-zéaralénol (Mirocha et coll., 1981), et de la toxine $T_{2}$ dont les formes majeures d'élimination sont 3 composés inconnus baptisés $\mathrm{TC}_{1}, \mathrm{TC}_{2}$ et $\mathrm{TC}_{6}$ (Yoshizawa et coll., 1981).

La quantité de mycotoxine rejetée dans le lait est toujours très faible par rapport à celle ingérée par la femelle laitière (Mirocha et coll., 1981 ; Ribelin, 1978 ; Yoshizawa et coll., 1981), dans le cas de l'aflatoxine, ce taux varie de 0,17 (Polan et coll., 1974) à 1-3\% (Masri et coll., 1969).

La récente revue bibliographique que nous avons effectuée (Tantaoui-Elaraki et coll., 1983) analyse les facteurs qui affectent ce taux d'élimination dans le cas des aflatoxines, et étudie la cinétique du rejet de l'aflatoxine $M_{1}\left(A_{F} M_{1}\right)$ dans le lait au cours et après l'ingestion de l'AFB $_{1}$.

Pour les fromages, cette forme de contamination indirecte n'a été étudiée, à notre connaissance, que pour l'AFM $\mathrm{M}_{1}$, sans doute parce qu'elle représente la forme majeure d'élimination des aflatoxines dans le lait (Afzal et coll., 1979) d'une part, et parce que sa toxicité et son pouvoir hépatocancérigène (Moreau, 1976; Applebaum et coll., 1982) attirent davantage l'attention des chercheurs d'autre part.

\subsection{Devenir de l'AFM ${ }_{1}$ au cours de la fabrication des fromages}

\subsubsection{Préparation du lait de fromagerie avant caillage}

- Effet de la réfrigération

Divers travaux ont montré la réduction du taux d'AFM $\mathrm{M}_{1}$ dans le lait stocké au froid. Mc Kinney et coll. (1977) observent une dispa-

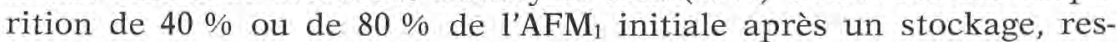
pectivement, de 4 ou de 6 jours à $0^{\circ} \mathrm{C}$. De même, Kiermeier et Mashaley (1977) observent une baisse de 11 à $25 \%$ du taux de l'AFM après 1 à 3 jours d'entreposage à $5^{\circ} \mathrm{C}$. Cependant, Stoloff et coll. (1975) notent une stabilité du taux de cette toxine dans le lait après 17 jours à $4^{\circ} \mathrm{C}$.

\section{- Effet de la pasteurisation}

La pasteurisation du lait n'entraîne pas la destruction totale de l'AFM $_{1}$ initialement présente (tab. 1).

Les effets obtenus peuvent être nuls ou tellement faibles qu'ils sont indétectables (Allcroft et Carnaghan, 1963 ; Stoloff et coll., 1975) ; ils peuvent aller jusqu'à une réduction de $64 \%$ (Purchase et coll., 1972). 
TABLEAU 1 - TABLE 1

Comportement de l'aflatoxine $\mathrm{M}_{1}$ au cours de la pasteurisation du lait

\begin{tabular}{|c|c|c|}
\hline $\begin{array}{c}\text { Barême } \\
\text { de } \\
\text { pasteurisation }\end{array}$ & $\begin{array}{l}\begin{array}{c}\text { Effet observé } \\
\mathrm{AFM}_{1} \text { détruite }\end{array} \\
\mathrm{AFM}_{1} \text { initiale }\end{array} 100$ & Références \\
\hline $62^{\circ} \mathrm{C}-30 \mathrm{~min}$ & 35 & KIERMEIER et MASHALEY (1977) \\
\hline $62^{\circ} \mathrm{C}-30 \mathrm{~min}$ & 32 & Purchase et coll. (1972) \\
\hline $62^{\circ} \mathrm{C}-30 \mathrm{~min}$ & Nul & STOLOFF et coll. (1975) \\
\hline $71^{\circ} \mathrm{C}-40 \mathrm{sec}$ & $28,9 \pm 11(a)$ & KIERMEIER et MASHALEY (1977) \\
\hline $71^{\circ} \mathrm{C}-40 \mathrm{sec}$ & 6 à 13 (b) & KIERMEIER et MASHALEY (1977) \\
\hline $72^{\circ} \mathrm{C}-45 \mathrm{sec}$ & 45 & Purchase et coll. (1972) \\
\hline $75^{\circ} \mathrm{C}-40 \mathrm{sec}$ & 12 & KIERMEIER et MASHALEy (1977) \\
\hline $77^{\circ} \mathrm{C}-16 \mathrm{sec}$ & Nul & StoLOFF et coll. (1975) \\
\hline $80^{\circ} \mathrm{C}-45 \mathrm{sec}$ & 64 & Purchase et coll. (1972) \\
\hline $80^{\circ} \mathrm{C}-45 \mathrm{sec}$ & Nul & Allcroft et CARNAGHaN (1963) \\
\hline $70^{\circ} \mathrm{C}-30 \mathrm{~min}$ & Nul & Allcroft et Carnaghan (1963) \\
\hline $74^{\circ} \mathrm{C}-30 \mathrm{~min}$ & 40 & KIERMEIER et MASHALEY (1977) \\
\hline
\end{tabular}

a : lait artificiellement contaminé.

b : lait naturellement contaminé.

Les divergences observées (tab. 1) peuvent s'expliquer par :

- la variabilité des températures appliquées, la réduction étant d'autant plus importante que la température est plus élevée (Purchase et coll., 1972) ;

- les méthodes d'analyse utilisées, notamment au niveau de l'extraction (Applebaum et coll., 1982) ;

- l'utilisation de lait naturellement ou artificiellement contaminé suivant les expériences (Applebaum et coll., 1982) l'AFM ${ }_{1}$ artificiellement additionnée semblant plus vulnérable aux traitements thermiques (Kiermeier et Mashaley, 1977). 


\section{- Effet de l'écrémage}

Certains fromages, dits maigres, sont préparés à partir de lait écrémé.

Au cours de l'écrémage, l'AFM 1 tend à rester préférentiellement dans le lait écrémé (Blanc et coll. 1983 ; Grant et Carlson, 1971 ; Kiermeier et Mashaley, 1977; Stoloff et coll., 1975). Dans le cas d'un lait naturellement contaminé, $84 \%$ de l'AFM ${ }_{1}$ initiale se retrouve dans le lait écrémé, cette proportion étant de 75 à $94,5 \%$ pour un lait artificiellement contaminé (Blanc et coll., 1983; Grant et Carlson, 1971; Kiermeier et Mashaley, 1977).

\subsubsection{Caillage, égouttage et travail du caillé}

\section{- Caillage du lait}

La fermentation lactique n'affecte pas la teneur initiale en $\mathrm{AFM}_{1}$ du lait ; bien au contraire, la quantité retrouvée dans le lait fermenté est légèrement plus élevée que celle du lait d'origine (Van Egmond et coll., 1977). Ce résultat s'explique probablement par une meilleure récupération de la toxine par les méthodes d'analyse après acidification.

Cette observation relative au yaourt peut, à notre avis, être étendue aux caillés lactiques de fromagerie.

Kiermeier et Buchner (1977 b) montrent que la quantité de présure utilisée pour le caillage du lait, ou la préparation de caillés par addition d'acides organiques n'entraînent aucun changement quant à la teneur en $\mathrm{AFM}_{1}$ et sa distribution par rapport à ce qui se passe dans le témoin.

Cependant, l'augmentation de la température au cours du caillage présure ou du caillage lactique se traduit par une baisse de la teneur en $\mathrm{AFM}_{1}$ du caillé (Kiermeier et Buchner, 1977 b).

\section{- Egouttage du caillé}

Les résultats concernant la répartition de $\mathrm{l}^{\prime} \mathrm{AFM}_{1}$ entre le lactosérum et le caillé de fromagerie sont très divergents. Certains auteurs retrouvent la totatlité de l'AFM ${ }_{1}$ dans le caillé (Allcroft et Carnaghan, 1963), d'autres, au contraire, la majeure partie de la toxine dans le lactosérum (Purchase et coll., 1972). Entre ces deux extrêmes, on trouve toutes sortes d'intermédiaires, avec la majeure partie de la toxine $(80 \%)$ dans le caillé (Mc Kinney et coll., 1973), ou la moitié de l'AFM 1 restant dans le lactosérum (Blanc et coll., 1983; Kiermeier et Mashaley, 1977; Stubblefield et Shannon, 1974) ou, enfin $86 \%$ dans le lactosérum et $14 \%$ dans le caillé (Stoloff et coll., 1975).

La divergence de ces résultats peut s'expliquer par la nature différente des fromages fabriqués et des modes de préparation, par l'influence des méthodes d'analyse (Mc Kinney et Cavanagh, 1977), ou par les conditions expérimentales telles que la forme naturelle ou arti- 
ficielle de la contamination en $\mathrm{AFM}_{1}$ du lait, et aussi le taux de contamination. Ainsi par exemple, Frémy et Roiland (1979) montrent, sur du lait cru additionné d'AFM $\mathrm{A}_{1}$, que la fabrication du Camembert aboutit à une répartition entre caillé et lactosérum qui dépend du taux de contamination initiale. En effet, plus le lait de départ est contaminé, plus la proportion restant dans le lactosérum semble élevée $(64,4 \%$ pour une contamination de $7,5 \mu \mathrm{g} / 1 ; 53 \%$ pour $3 \mu \mathrm{g} / 1$; et $42,3 \%$ pour $0,3 \mu \mathrm{g} / \mathrm{l})$.

Suite à la perte de toxine dans le lactosérum, la quantité totale d'AFM $M_{1}$ récupérable dans un fromage est plus faible que celle présente initialement dans le lait. Cependant, le pourcentage de réduction de cette quantité récupérable varie, suivant les auteurs, de $20 \%$ (Stoloff et coll., 1975) à près de $65 \%$ (Purchase et coll., 1972).

Toutefois, la concentration en $\mathrm{AFM}_{1}$ du fromage est toujours supérieure à celle du lait initial, le facteur de multiplication variant de 1,7 à 8,1 (tab. 2). Ceci est en accord avec la suggestion de Grant et Carlson (1971) selon laquelle le taux d'AFM d $_{1}$ après les opérations de séparation dans l'industrie laitière est lié à la teneur en caséine et matière sèche des produits obtenus. Le récent article de Blanc et coll. (1983) apporte de nouveaux arguments en faveur de la théorie, avancée par de nombreux chercheurs, de la fixation de l'AFM $_{1}$ sur les protéines du lait.

\section{TABLEAU $2-T A B L E 2$}

Taux de concentration de l'aflatoxine $M_{1}$ dans les fromages par rapport à celle du lait initial :

Concentration de l'aflatoxine $\mathbf{M}_{1}$ dans le fromage

concentration de l'aflatoxine $\mathbf{M}_{1}$ dans le lait

\begin{tabular}{l|c|c}
\hline \multicolumn{1}{c|}{ Fromages } & Taux de concentration & \multicolumn{1}{c}{ Références } \\
\cline { 1 - 2 } Brick & 1,7 & BRACKETT et coll. (1982) \\
Fromage frais & 3,2 & KIERMEIER et BUCHNER (19T7 b) \\
Tilsit & 3,7 & KIERMEIER et BUCHNER (1977 b) \\
Camembert & 3,7 & KIERMEIER et BUCHNER (1977 b) \\
Parmesan & 5,8 & BRACKETT et MARTH (1982 b) \\
Mozzarella & 8,1 & BRACKETT et MARTH (1982 b) \\
\hline
\end{tabular}


- Travail du caillé

Le lavage du caillé, dans des conditions de fabrication normale peut entraîner l'élimination d'une partie de l'AFM $\mathbf{M}_{1}$, allant jusqu'à $22 \%$ de sa teneur initiale (Kiermeier et Buchner, 1977 b). Un lavage

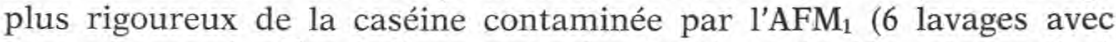
des volumes de $40 \mathrm{ml}$ d'eau pour des portions de $10 \mathrm{~g}$ de caséine) a permis à Blanc et coll. (1983) d'obtenir une réduction de près de $97 \%$ du taux initial de toxine.

De même, le salage en saumure du caillé de Camembert peut permettre l'élimination d'une faible proportion (6\% de la quantité totale de départ) dans la saumure (Frémy et Roiland, 1979).

Par ailleurs, le chauffage du caillé de fromagerie à $80^{\circ} \mathrm{C}$ pendant 5 min (Kiermeier et Weiss, 1976) ne semble entraîner aucune réduction de l'AFM 1 , initialement présente.

\subsubsection{PréParation des fromages fondus}

Le fromage fondu fabriqué à partir de Cheddar, lui-même préparé avec du lait naturellement contaminé, contient autant d'AFM $\mathbf{M}_{1}$ que le fromage d'origine (Brackett et Marth, 1982 a). De plus, le traitement thermique $\left(90^{\circ} \mathrm{C}, 20 \mathrm{~min}\right)$ ou l'addition du sel émulsifiant $\left(5 \% \mathrm{Na}_{2} \mathrm{HPO}_{4}\right)$ ou les 2 traitements combinés semblent entraîner une élévation de la teneur d'AFM 1 récupérable (Brackett et Marth 1982 a).

Polzhofer (1977 b) avait, lui, trouvé une baisse de $9 \%$ de l'AFM au cours de la fabrication du fromage fondu. Cependant, cet auteur avait utilisé un Emmenthal artificiellement contaminé.

\section{CONTAMINATION DIRECTE : LES MOISISSURES TOXINOGENES DANS LES FROMAGES}

\subsection{Moisissures spécifiques des fromages et susceptibles d'être toxinogènes}

La fabrication des fromages bleus fait intervenir des souches de Penicillium roqueforti qui leur donnent leur marbrure verdâtre caractéristique et participent pour une large part à leur affinage et au développement de leur arôme.

En 1973, Wei et coll. mettent en évidence la PR toxine dans les filtrats de culture de P.roqueforti. Cependant, d'autres auteurs, dont Guiraud et coll. (1977) montrent, d'une part, qu'il existe des souches toxinogènes et d'autres incapables de produire la PR toxine, et d'autre part, que la synthèse de cette toxine ne se produit que dans des conditions particulières du milieu (15\% de saccharose), très éloignées de celles qui existent dans les fromages. En outre, selon Engel et 
Prokopek (1979), ce sont surtout les souches isolées sur d'autres aliments que les fromages qui se révèlent productrices de PR toxine.

Plus tard, Lafont et coll. (1979 a) ont montré la production, au laboratoire, d'acide mycophénolique par différentes souches de P.roqueforti isolées de fromages. Mais sur 62 souches étudiées par Engel et coll. (1982), seulement 7 étaient capables de produire cette toxine, et elles avaient toutes été isolées sur des fromages provenant du même fabricant.

Ce même P.roqueforti peut synthétiser d'autres mycotoxines : l'acide pénicillique et la patuline (Moreau, 1980; Olivigni et Bullerman, 1977), la roquefortine (Engel et Prokopek, 1979; Ohmono et coll. 1979), la festuclavine (Ohmono et coll., 1979) et les isofumigaclavines A et B (Scott et coll., 1976).

Cependant, une étude menée par Teuber et Engel (1983) sur de nombreuses souches industrielles de P.roqueforti a montré qu'elles ne synthétisaient aucune des mycotoxines suivantes : aflatoxines, citréoviridine, citrinine, diacetoxyscirpénol, ochratoxine A, patuline, acide pénicillique, rubratoxine $B$, stérigmatocystine, trémortine $A$ et zéaralénone. Ce résultat confirme ceux de Frank et coll. (1977) selon lesquels l'ingestion de mycélium de P.roqueforti n'induisait ni le cancer ni aucun autre effet lésionnel sur les organes chez la truite et le rat.

En revanche Lafont et coll. (1976), étudiant la toxicité in vitro des extraits de culture de 50 souches de Penicillium utilisés dans la maturation des fromages à pâte bleue et à pâte molle observent que la moitié des souches étudiées provoquent des effets toxiques variables sur les embryons de poulets, les souris et les cultures de cellules de rats : les extraits de deux souches ont causé la mort rapide chez la souris après injection.

Une autre expérience menée par Gibel et coll. (1971), qui n'a cependant jamais été reproduite, aurait montré que les extraits de culture d'une moisissure, bizarrement dénommée P.camemberti var. candidum, auraient provoqué un effet oncogène chez le rat Wistar.

Dans une investigation de Lebars (1979 a), la totalité des souches de P.camemberti isolées à partir de 20 échantillons de fromages se sont révélées capables de produire de l'acide cyclopiazonique sur des milieux de laboratoire. Confirmant ce résultat, Engel (1981) a observé que les 62 souches étudiées de P.camemberti et de P.caseicolum étaient capables de synthétiser cette toxine au laboratoire.

Teuber et Engel (1983), rapportent cependant les résultats, plus rassurants, d'une étude qui a porté sur environ 40 souches de P.camenberti et P.caseicolum isolées de fromages et d'inoculums industriels : toutes ces souches étaient incapables de produire, dans des conditions 
optimales, les mycotoxines suivantes : aflatoxines $B_{1}, B_{2}, G_{1}, G_{2}$ et $M_{1}$, citréoviridine, citrinine, diacetoxyscirpénol, ochratoxine $\mathrm{A}$, patuline, acide pénicillique, PR toxine, rubratoxine $B$, stérigmatocystine, trémortine A, et zéaralénone. En outre, les extraits de cultures se sont montrés inoffensifs dans un certain nombre de tests biologiques.

\subsection{Flore toxinogène indésirable des fromages}

La contamination du lait par des spores de moisissures diverses peut avoir lieu au cours de la traite (Guéguen et coll., 1978; Kiermeier, 1974) ou par l'intermédiaire des ustensiles (Frank, 1974). Cette contamination peut avoir lieu aux stades plus avancés de la préparation du caillé, de l'affinage et du stockage au froid (Bullerman et Olivigni, 1974). L'introduction d'épices et fines herbes dans certains fromages peut également être une source appréciable de contamination. Tel est le cas du poivre, souvent pollué par les Aspergillus producteurs d'aflatoxines (Jacquet et Téhérani, 1974).

Pour peu que ces moisissures trouvent des conditions favorables, elles se développent et libèrent, s'il s'agit de souches toxinogènes, des mycotoxines dans le fromage. Deux séries de recherches sont menées dans ce contexte :

- celle qui consiste à isoler et à identifier les souches de moisissures qui contaminent les fromages, et, dans certains cas, à déterminer le pouvoir toxinogène de ces moisissures en les cultivant sur des milieux de laboratoire favorables à la toxinogénèse ;

- celle qui consiste à utiliser les fromages comme milieux de culture pour des espèces fongiques toxinogènes afin de déterminer l'aptitude de ces dernières à excréter leurs métabolites toxiques dans ces produits.

\section{FROMAGES \\ 2.2.1. NATURE DE LA MYCOFLORE TOXINOGÈNE INDÉSIRALE DES}

Les fromages sujets à la contamination par les moisissures indésirables sont surtout ceux qui ne comportent pas de moisissures spécifiques.

La plupart du temps, ce sont les Penicillium qui occasionnent ces contaminations (Bullerman, $1976 \mathrm{a}, 1977 \mathrm{~b}, 1980$; Bullerman et Olivigni, 1974 ; Jarvis, 1983 ; Lafont et coll., 1979 c ; Northolt et coll., 1980; Torrey et Marth, 1977). Ainsi, dans un travail de Bullerman et Olivigni (1974) sur le Cheddar, 349 souches de moisissures ont été isolées dont $82,2 \%$ appartenaient à ce genre, les espèces d'Aspergillus et de Fusarium représentant, respectivement, 6,6 et 1,1\%. Parmi les souches isolées, 29,2\% donnaient lieu à des cultures dont les extraits étaient toxiques pour l'embryon de poulet. Ce pourcentage était de $29,5 \%$ pour les Penicillium et de 47,8\% pour les Aspergillus. 
D'autre part, des mycotoxines connues ont été trouvées dans $7,2 \%$ des extraits de cultures; il s'agissait de la patuline, de l'acide pénicillique, de l'ochratoxine A et des aflatoxines.

Des résultats analogues ont été observés par Bullerman (1976 a) qui a isolé à partir de fromages suisses, 183 souches de moisissures dont $87 \%$ appartenaient au genre Penicillium. $34 \%$ de l'ensemble des isolats, et $35 \%$ des Penicillium, se sont montrés toxiques pour l'embryon de poulet. Des mycotoxines connues ont été détectées dans $5,50 \%$ des extraits, où l'on a identifié de l'acide pénicillique, de la patuline et de l'aflatoxine.

Une analyse similaire a été menée par Lafont et coll. (1979 c) sur 235 échantillons de fromages de types variés. Ces auteurs ont pu identifier plusieurs souches de P.expansum, P.urticae, P.cyclopium, P.palitans, P.brevicompactum et P.viridicatum; et la plupart de ces souches se sont montrées toxinogènes in vitro, produisant la patuline, l'acide pénicillique, ou l'acide mycophénolique. A côté des Pénicillium, 2 souches d'A.versicolor et une d'A.sydowi ont été isolées à partir de fromages à pâte ferme et se sont révélées capables de produire de la stérigmatocystine.

Un travail plus récent de Jarvis (1983), a mis en évidence la prédominance des espèces de Penicillium de la série Assymetrica. Cet auteur identifie notamment P.roqueforti, P.griseofulvum, P.chrysogenum, P.expansum, P.cyclopium, P.crustosum, P.citrinum, P,aurantiogriseum, P.brevicompactum et P.viridicatum.

P.crustosum a aussi été isolé, à partir d'un fromage moisi, par Arp et Richard (1978) qui ont montré qu'il était capable de produire une mycotoxine, le pénitrème $\mathrm{A}$.

Signalons également cet accident de fabrication étudié par Guéguen et coll. (1978) qui ont identifié le P.roqueforti comme contaminant dans le Camembert et le Carré d'Auge.

Mais malgré la diversité des espèces rencontrées, P.cyclopium reste prédominant en général, suivi de P.viridicatum (Bullerman, 1980; Northolt et coll., 1980).

Bien que moins fréquents que les Penicillium, les Aspergillus ont souvent été isolés comme contaminants naturels des fromages. A.flavus, producteur d'aflatoxine, a notamment été signalé (Bullerman, 1976 a, 1980). De même, Lafont et coll. (1979 c) ont isolé sur des fromages à pâte ferme des souches d'A.versicolor et d'A.sydowi productrices de stérigmatocystine. Enfin, Northolt et coll. (1980) ont également détecté A.versicolor et A.repens, mais la toxinogénèse des souches isolées n'a pas été vérifiée.

Enfin, signalons que les autres champignons isolés appartiennent notamment aux genres Fusarium (Bullerman, 1976 b, 1980 ; Bullerman et Olivigni, 1974) Cladosporium (Bullerman, 1976 b, 1980) et Alternaria (Bullerman et Olivigni, 1974). 


\subsubsection{TOXINOGÉNÈSE DES MOISISSURES SUR LES FROMAGES}

Un certain nombre de recherches ont été conduites pour savoir si les moisissures toxinogènes sont susceptibles d'excréter leurs toxines dans les fromages. Il est bien évident que de nombreux facteurs interviennent dans la synthèse de ces mycotoxines, dont essentiellement les conditions physico-chimiques et biologiques liées au substrat (c'est-à-dire le fromage concerné), la température, la composition de l'atmosphère, en plus d'autres facteurs tels que l'utilisation d'enrobage inerte (paraffines), etc.

\section{- Facteurs nutritionnels}

Le lait entier stérilisé est un milieu très favorable à la croissance de l'A.flavus et à la production d'aflatoxine (Boutibonnes et Jacquet, 1967). La crème est également un excellent substrat et, additionnée au lait entier, elle le rend encore plus propice à la toxinogénèse (Jacquet et Boutibonnes, 1970). D'autre part, Lie et Marth (1968) ont montré que les substrats à base de caséine étaient favorables à la production d'aflatoxines $B_{1}$ et $G_{1}$ par A.flavus et A.parasiticus.

Quant au lactosérum, bien que permettant une assez bonne croissance d'A.flavus (Jacquet et Tantaoui-Elaraki, 1975), il n'est pas favorable à la synthèse de l'aflatoxine. D'ailleurs, quand le lactose est utilisé comme seule source de carbone, il ne permet qu'une croissance très faible (Davis et coll., 1967; Jacquet et Tantaoui-Elaraki, 1975 ; Mateles et Adye, 1965) avec une production d'aflatoxine nulle (Jacquet et Tantaoui-Elaraki, 1975) ou infime (Davis et coll., 1967; Mateles et Adye, 1965).

Ainsi, les fromages, formés essentiellement de caséine et de matière grasse, et pratiquement dépourvus de lactose, constituent, sur le plan nutritionnel du moins, des substrats favarables à la croissance des Aspergillus et à la synthèse des aflatoxines. En effet, cultivant des souches toxinogènes d'A.flavus ou d'A.parasiticus, différents auteurs ont obtenu des productions variables d'aflatoxines sur Cheddar (Lie et Marth, 1967 ; Oldham et coll., 1971), Brick, Tilsit (Engel, 1978; Frank, 1967; Kiermeier et Groll, 1970), Emmenthal (Jacquet et Tantaoui-Elaraki, 1975) de même que sur Mozzarella (Lieu et Bullerman, 1977), sur "Bonbel», Gouda, fromage des Pyrénées, "Vachequirit» (fromage fondu) et "Boursin ail et fines herbes 》 (fromage non affiné) (Jacquet et Tantaoui-Elaraki, 1975). Les quantités d'aflatoxines sont parfois très élevées : $49 \mathrm{mg} / \mathrm{kg}$ sur le Cheddar (Lie et Marth, 1967), $97,5 \mathrm{mg} / \mathrm{kg}$ sur le Gouda et $107,25 \mathrm{mg} / \mathrm{kg}$ sur l'Emmenthal (Jacquet et Tantaoui-Elaraki, 1975).

D'autre part, les travaux de Lafont et coll. (1979 c) suggèrent que divers fromages constituent un milieu où est possible la synthèse de stérigmatocystique, de patuline, d'acide pénicillique et d'acide mycophénolique : ces auteurs ont montré un lien entre, d'une part, 
la présence de telles mycotoxines dans les fromages, et d'autre part, la présence dans les mêmes fromages de moisissures capables d'excréter ces mycotoxines sur des milieux de laboratoire.

En effet, un Penicillium producteur d'ochratoxine A s'est révélé capable d'en produire sur Cheddar après 3 semaines à $25^{\circ} \mathrm{C}$, mais beaucoup moins que dans les substrats, riches en carbohydrates (Polhmeier et Bullerman, 1978). De même, Stott et Bullerman (1976) ont montré que P.patulum peut croître sur le fromage Cheddar, mais la production de patuline est nulle ou très faible.

Les fromages peuvent également constituer un milieu favorable à la production de pénitrème A par P.crustosum, tel que l'ont montré Richard et Arp (1979).

Enfin signalons cette investigation menée par Engel (1978) selon laquelle le Tilsit constitue un milieu peu favorable à la synthèse d'un certain nombre de mycotoxines : les aflatoxines $B_{1}, B_{2}, G_{1}$ et $M_{1}$ par A.flavus ou A.parasiticus, la citrinine par P.citrinum, l'ochratoxine par A.ochraceus, la patuline, l'acide pénicillique et la PR toxine par P.roqueforti et, enfin, la stérigmatocystine par A.versicolor et A.nidulans. Seule la citréoviridine semble produite en quantité relativement importante.

Selon plusieurs auteurs, l'absence de production d'acide pénicillique et de patuline, et la synthèse limitée des aflatoxines et de l'ochratoxine A sur les fromages seraient dues à la faible teneur en carbohydrates et la richesse élevée en protéines (Frank, 1968; Lieu et Bullerman, 1977 ; Olivigni et Bullerman, 1977 ; Stott et Bullerman, 1975 ; Shih et Marth, 1972 a).

\section{- Facteurs physiques}

La température : la plupart des souches de champignons isolées des fromages se révèlent psychrotrophes (Bullerman, 1977 a). D'ailleurs, Bullerman (1976 a) observe que, même conservés à $5^{\circ} \mathrm{C}$, tous les échantillons de fromage suisse montrent un développement visible de moisissure après 5 semaines. Toutefois, un effet de sélection peut être opéré par la température d'entreposage; dans les fromages réfrigérés, par exemple, seuls les Penicillium sont à craindre (Bullerman et Olivigni, 1974).

Certaines moisissures sont capables de produire des mycotoxines à des températures très basses allant de $-2^{\circ}$ à $10^{\circ} \mathrm{C}$ (Bullerman, 1979). La synthèse de l'aflatoxine, cependant, nécessite des températures plus élevées. Kiermeier et Groll (1970) ont montré que la croissance des Aspergillus toxinogènes sur le Tilsit se produit entre les limites de 18 et $36^{\circ} \mathrm{C}$, avec un optimum à $26^{\circ} \mathrm{C}$. De même Oldham et coll. (1971) observent que A.flavus produit de l'aflatoxine, quoique faiblement, sur du Cheddar à $25^{\circ} \mathrm{C}$, mais pas à 4,4 ou à $7,2^{\circ} \mathrm{C}$. Shih et Marth (1972 a) confirment que A.flavus et A.parasiticus sont capables de croître sur le fromage Brick à 7,$2 ; 12$ et $23,9^{\circ} \mathrm{C}$; cepen- 
dant l'A.flavus ne produit de $1^{\prime}$ aflatoxine qu'à $23,9^{\circ} \mathrm{C}$, alors que $1^{\prime}$ A.parasiticus en produit à 12 et à $23,9^{\circ} \mathrm{C}$.

Stott et Bullerman (1976) observent des résultats comparables concernant la synthèse de patuline, possible à $25^{\circ} \mathrm{C}$ sur Cheddar ensemencé avec P.patulum, mais pas à $5^{\circ} \mathrm{C}$, malgré une bonne croissance de la moisissure.

Dans les conditions naturelles de contamination, c'est la croissance des Penicillium non toxinogènes qui semble prédominer aux basses températures, ce qui réduit considérablement les risques d'accumulation des toxines dans les fromages. Le froid permet également d'éviter le développement des Aspergillus, notamment A.flavus et A.parasiticus producteurs d'aflatoxine, et d'A.versicolor producteur de stérigmatocystine (Bullerman, 1981), d'où l'intérêt de maintenir les fromages au froid autant que possible.

L'humidité : celle du substrat, ou plus précisément, l'activité de l'eau (aw) a un rôle primordial. L'aw optimale pour la croissance d'A.flavus est supérieure à 0,93 et le minimum est de 0,78 (Jarvis, 1971). Sur des tranches de Tilsit, Kiermeier et Groll (1970) ont observé que la toxinogénèse exigeait un minimum de $72 \%$ d'humidité relative (teneur en humidité du fromage : environ $45 \%$ ). Applebaum et coll., (1982) font observer, en conséquence, que les fromages peuvent être plus ou moins exposés à la contamination par l'aflatoxine du fait de l'humidité différente de l'un à l'autre, et rien ne nous empêche d'étendre cette remarque aux autres mycotoxines.

La faible activité de l'eau pourrait également être à l'origine de la production nulle ou faible de la patuline (Northolt et coll., 1978), de l'acide pénicillique (Northolt et coll., 1979a) et de l'ochratoxine (Northolt et coll., $1979 \mathrm{~b}$ ) dans les fromages.

Le $\mathrm{pH}$ :

Lié et Marth (1968) ont montré que la synthèse d'aflatoxine sur des substrats à base de caséine était très bonne aussi bien aux $\mathrm{pH}$ acides qu'aux $\mathrm{pH}$ alcalins.

Cependant, d'après les données de plusieurs études rapportées par Kiermeier (1974), un $\mathrm{pH}$ de 4,5 est favorable à la production d'aflatoxine, et celle-ci décroît nettement aux pH voisins de 7 .

Applebaum et coll. (1982) font remarquer que ces zones de $\mathrm{pH}$ favorables coïncident avec les valeurs qui se rencontrent généralement dans certains fromages non affinés et que la contamination par l'aflatoxine devrait se faire plus facilement au début de la maturation. On pourrait rapprocher de ces remarques les observations de Jacquet et Tantaoui-Elaraki (1976) relatives aux modifications de comportement de la pâte du fromage Camembert, au cours de son affinage, vis-à-vis des spores d'A.flavus inoculées artificiellement. Ces auteurs ont, en effet, observé que les parties centrales du fromage, peu favorables à la croissance au premier jour de fabrication, permettaient une bonne croissance si le fromage était plus âgé. Par 
contre, la partie superficielle qui, au premier jour, ne se distingue pas du reste du fromage, devient de plus en plus réfractaire à la croissance de l'A.flavus.

Mais il y a certainement d'autres phénomènes qui concourent à cette évolution, tels que les compétitions microbiennes par exemple.

\section{- Facteurs biologiques}

Les ferments lactiques semblent avoir peu d'effet sur les Aspergillus producteurs d'aflatoxines. Ainsi, Lactobacillus casei n'a pas d'effet sur la croissance d'A.parasiticus quand les 2 micro-organismes sont ensemencés simultanément, mais entraîne une réduction de toxinogénèse (Elgendy et Marth, 1981). Une réduction de croissance était cependant obtenue, et la toxinogénèse était encore plus faible, si la bactérie était ensemencée dans le milieu 3 jours avant la moisissure.

En revanche, la culture de l'A.parasiticus en présence de Streptococcus lactis améliore la croissance de la moisissure et augmente la quantité d'aflatoxine produite. Ce dernier effet n'est cependant observé que si les 2 micro-organismes sont ensemencés en même temps (Wiseman et Marth, 1981).

Le Brevibacterium linens, impliqué dans l'affinage de certains fromages, exerce une légère inhibition de la croissance et de la toxinogénèse d'A.parasiticus quand les 2 micro-organismes croissent ensemble (Weckbach et Marth, 1977), ce qui corrobore les observations de Shih et Marth (1972 a) selon lesquelles le Brick, fromage affiné avec cette bactérie, est relativement peu favorable à la production d'aflatoxine.

D'autre part, la morge (couche visqueuse constituée de plusieurs micro-organismes) qui se développe sur le fromage Romadur serait la raison pour laquelle les Aspergillus producteurs d'aflatoxine sont incapables de se développer sur ce fromage (Kiermeier et Groll, 1970).

Mais les effets de compétition les plus nets sont certainement ceux dus aux moisissures spécifiques des fromages. Ainsi, Kiermeier et Groll (1970) observent que les moisissures productrices d'aflatoxine ne se développent pas sur le Camembert, et que le Penicillium caseicolum, agent de maturation de ce fromage, inhibe la production d'aflatoxine par ces micro-organismes. Les travaux de Jacquet et Tantaoui-Elaraki (1976) montrent que ces observatoins sont généralisables à d'autres fromages à moisissure externe (Camembert, Brie, Coulommiers, Caprice-des-Dieux, fromage de chèvre) ou interne (Bleu d'Auvergne et Roquefort). En effet, tous ces fromages étaient réfractaires au développement d'A.flavus et d'A.parasiticus, mais l'ensemencement de la partie centrale seule des fromages à croute moisie permettait souvent une croissance intense et parfois une forte production d'aflatoxine $(331 \mathrm{mg}$ d'aflatoxine par $\mathrm{kg}$ sur le fromage de chèvre sans croûte). Aucune substance inhibitrice des Aspergillus 
n'a été isolée par ces auteurs, mais ils ont montré que le chauffage d'une pâte de Camembert entier pendant $20 \mathrm{~min}$ à une température supérieure ou égale à $60^{\circ} \mathrm{C}$ la rendait favorable à la croissance d'A.flavus, et qu'un chauffage de 100 à $120^{\circ} \mathrm{C}$ pendant le même temps la rendait propice à la sécrétion de petites quantités d'aflatoxine, alors que même un chauffage à $120^{\circ} \mathrm{C}$ pendant $20 \mathrm{~min}$ laissait le Roquefort et le Bleu d'Auvergne toujours défavorables à la croissance d'A.flavus.

\section{Autres facteurs}

Substances antimicrobiennes : l'acide sorbique, antifongique le plus utilisé dans les fromages (Applebaum et coll., 1982), provoque une perte de viabilité des spores d'A.parasiticus (Przybylski et Bullerman, 1980), mais ne semble pas affecter l'aptitude de cette espèce à produire l'aflatoxine, bien que réduisant sa croissance (Youssef et Marth, 1981). Les sorbates retardent la croissance des champignons isolés des fromages (Bullerman, 1977 a, 1977 b), mais la plupart des souches sont capables de croître en présence de $0,3 \%$ de sorbate de potassium à $5^{\circ} \mathrm{C}$ après 7 jours d'incubation. Cependant, Stott et Bullerman (1976) observent l'inhibition de croissance du P.patulum par le sorbate de potassium sur le Cheddar.

Les propionates sont utilisés, aux concentrations finales de 0,1 à $0,3 \%$ dans les fromages (Bullerman, $1977 \mathrm{~b}$ ). Mais l'action, fongistatique, des propionates comme des sorbates, n'est effective que si le taux de contamination des fromages en spores n'est pas trop élevé.

La pimaricine est également employée contre les moisissures de surface des fromages (Bullerman, 1977 a; Morris et Catsberg, 1980 ; Morris et Hart, 1978). Son efficacité contre les champignons isolés des fromages est cependant plus élevée à 5 et $12^{\circ} \mathrm{C}$ qu'à $25^{\circ} \mathrm{C}$ (Bullerman, 1977 a). D'autre part, elle n'a d'efficacité que si elle est utilisée avant le début de croissance des moisissures, et si celle-ci a lieu, la pimaricine ne stoppe pas la production d'aflatoxine (Kiermeier et Zierer, 1975). Pourtant, Shahani et Godberg (1972) avaient montré une nette réduction de la croissance mycélienne et de la toxinogénèse d'A.flavus par la pimaricine.

Jacquet et Tantaoui-Elaraki (1976) ont observé la possibilité d'A.flavus de croître sur le fromage de Pont-l'Evêque dépourvu de sa croûte, alors que sur le fromage entier, cette croissance était inhibée. Ils ont ensuite démontré que la surface de ce fromage et le papier d'emballage contenaient une substance antifongique, qui n'a pas été identifiée.

Enfin, le sel ajouté au cours de la fabrication des fromages peut avoir un rôle inhibiteur sur les moisissures de contamination ; par exemple, Shih et Marth (1972 b) ont montré qu'une concentration en $\mathrm{NaCl}$ de 1 à $3 \%$ augmente la production d'aflatoxine et qu'au delà de $3 \%$, le sel réduit cette synthèse. 
Cire d'enrobage et croûte sèche : Stott et Bullerman (1976) ont montré le rôle protecteur de l'enrobage dans de la cire, pratique courante pour certains fromages; et ce rôle pourrait aussi être joué, d'après Applebaum et coll. (1982), par la croûte sèche et dure que présentent d'autres fromages.

Le conditionnement des fromages : la composition de l'atmosphère influence considérablement la croissance des moisissures et la toxinogénèse. La production d'aflatoxine, en particulier, est inhibée par les concentrations élevées en $\mathrm{CO}_{2}$ (Landers et coll., 1967) et dans les atmosphères à concentration élevée en azote et diminuée en $\mathrm{O}_{2}$ (Shih et Marth, 1973) ; et Applebaum et coll., (1982) suggèrent que le contrôle de la composition de l'atmosphère dans les salles d'affinage ou dans les emballages pourrait prévenir la croissance des moisissures sur les fromages. Effectivement, Stott et Bullerman (1976) ont montré que l'emballage sous vide du fromage Cheddar empêche la croissance du P.patulum à 5 et à $25^{\circ} \mathrm{C}$.

\section{STABILITE DES MYCOTOXINES DANS LES FROMAGES}

\subsection{Devenir de $\mathrm{I}^{\prime} \mathrm{AFM} \mathrm{M}_{1}$ au cours de l'affinage et du stockage des fromages}

L'AFM $_{1}$ dans les fromages est assez stable, dans l'ensemble. Cette stabilité dure au moins 3 mois d'après Kiermeier et Buchner (1977 b). D'autres auteurs ont noté des durées de stabilité plus longues : 17 semaines dans le Mozzarella (Brackett et Marth, 1982 b) et 26 semaines dans le Brick et le fromage de type Limburger (Brackett et coll., 1982).

Cependant, à part Frémy et Roiland (1979) qui observent une diminution continuelle du taux d'AFM $\mathrm{d}_{1}$ dans le Camembert au cours du temps, la plupart des auteurs notent une évolution irrégulière (Brackett et coll., 1982 ; Brackett et Marth, 1982 a, 1982 b ; Kiermeier et Buchner, 1977 b).

Plusieurs hypothèses ont été émises pour expliquer ces phénomènes. D'abord, les conditions physico-chimiques du fromage étant changeantes au cours de l'affinage, elles influenceraient le taux de récupération des méthodes d'analyse (Brackett et Marth, 1982 a). De même, la protéolyse limitée, due à l'activité microbienne, aurait pour conséquence de rendre l'AFM ${ }_{1}$ plus accessible aux solvants d'extraction, cependant que la libération d'ammoniac, conséquence de cette protéolyse à un stade plus avancé, entraînerait la destruction d'une partie de la toxine (Brackett et coll., 1982). Enfin, une partie de ces variations, notamment celles observées à la périphérie des fromages, 
peut s'expliquer par la perte d'eau au cours du stockage (Brackett et coll., 1982; Kiermeier et Buchner, 1977 b).

\subsection{Stabilité des autres mycotoxines dans les fromages}

Les aflatoxines $B_{1}$ et $G_{1}$ sont également stables dans les fromages. 90 à $100 \%$ des quantités initiales étaient encore présentes après un stockage d'une semaine à $5^{\circ} \mathrm{C}$ dans une expérience de Lieu et Bullerman (1977).

Cette stabilité relative des aflatoxines contraste avec l'instabilité d'autres mycotoxines. En effet, Stott et Bullerman (1976) notent une disparition très rapide (plus de $73 \%$ en $3 \mathrm{~h}$ ) de la patuline dans le Cheddar artificiellement contaminé $(5 \mu \mathrm{g} / \mathrm{g})$ et stocké à 5 ou $25^{\circ} \mathrm{C}$. De même, Lieu et Bullerman (1977) observent que seule une faible teneur de patuline ou d'acide pénicillique ( 5 à $8 \%$ de la teneur initiale) persiste dans le fromage suisse après une semaine à $5^{\circ} \mathrm{C}$. La PR toxine est également rapidement inactivée dans les fromages (Engel et Prokopek, 1979).

En revanche, l'ochratoxine semble généralement plus stable dans les aliments que la patuline et l'acide pénicillique, mais probablement moins que les aflatoxines (Bullerman, 1981). Polhmeier et Bullerman (1978), en effet, ont observé que $49 \%$ de l'ochratoxine ajoutée au Cheddar étaient encore présents après un stockage de 48 h à $25^{\circ} \mathrm{C}$.

\section{PRESENCE DE MYCOTOXINES DANS LES FROMAGES DU COMMERCE}

Comme nous venons de le voir, dans les fromages on peut trouver non seulement les métabolites toxiques provenant du lait après ingestion d'aliments contaminés par les femelles laitières (cas de l'AFM $M_{1}$ ) mais également des toxines synthétisées par des moisissures spécifiques ou de contamination.

\subsection{Cas de l'aflatoxine $M_{1}$}

Les analyses effectuées en R.F.A. entre 1972 et 1974 (Polzhofer, 1977 a) et en 1976 (Kiermeier et Buchner, 1977 a) indiquent une fréquence très élevée de contamination dans tous les types de fromages, Polzhofer (1977 a) ayant noté un taux de contamination compris entre 0,10 et $1,30 \mu \mathrm{g} / \mathrm{kg}$. Les analyses effectuées en France donnent des résultats plus hétérogènes ; ceux de Corbion et Frémy (1978) indiquent seulement un échantillon contaminé (4 ppb) sur 100 Camemberts analysés, alors que Blanc et Karleskind (1981) trouvent une fréquence de contamination de $30 \%$ environ pour les fromages frais et affinés. 
Les analyses effectuées par Stolof et coll., (1981) semblent indiquer que les risques de contamination sont négligeables aux U.S.A. en année normale. En effet, sur 190 prélèvements de Cheddar et 209 de " Cottage cheese » effectués en 1979, ces auteurs détectent seulement un échantillon de ce dernier type contaminé avec $0,3 \mathrm{ng} / \mathrm{g}$.

\subsection{Cas des autres mycotoxines}

Le tableau 3 résume les résultats de certaines analyses de fromages pour la recherche de mycotoxines susceptibles d'avoir été produites sur les fromages suite au développement de moisissures, normales ou indésirables.

La roquefortine et les isofumigaclavines $\mathrm{A}$ et $\mathrm{B}$ ont été trouvées dans des fromages à pâte persillée (Scott et Kennedy, 1976). De même dans un travail de Ware et coll. (1980), tous les échantillons de fromages bleus et de sauces de fromages bleus analysés s'avéraient positifs pour la recherche de la roquefortine, mycotoxine produite par le P.roqueforti agent d'affinage de ces fromages. Cependant, les teneurs de roquefortine trouvées sont généralement faibles, avec un maximum de $6,8 \mathrm{mg} / \mathrm{kg}$ de fromage (Engel et Prokopek, 1979).

L'acide mycophénolique peut également contaminer les fromages affinés avec P.roqueforti (Engel et coll., 1982), mais les teneurs observées sont très en-deça de celles pouvant inquiéter la santé du consommateur.

D'autre part, et bien que le fromage ne semble pas fournir au P.roqueforti des conditions favorables à la synthèse de la PR toxine (Guiraud et coll., 1977), certains auteurs (Jacquet et coll., 1981) $n^{y}$ excluent pas sa présence dans les produits du commerce.

Dans les fromages de type "Camembert », l'acide cyclopiazonique a été détecté en France (Le Bars, 1979a) et en Allemagne (Engel, 1981), mais beaucoup plus dans la croûte que dans la pâte des fromages, et à des taux qui ne suscitent aucune inquiétude pour la santé du consommateur.

D'autre part, les travaux de Lafont et coll. $(1976,1979$ b, 1979 c) démontrent que la stérigmatocystine peut contaminer les fromages à pâte dure, alors que la patuline, l'acide pénicillique et l'acide myophénolique se rencontrent dans divers types de fromages. Ces auteurs (Lafont et coll., 1976, 1979 b) montrent clairement qu'il existe une corrélation entre la présence de ces mycotoxines dans les fromages et la présence sur les mêmes fromages de moisissures capables de produire ces toxines au labaratoire. Northolt et coll. (1980) confirment la présence de stérigmatocystine dans les fromages hollandais, à des taux allant de 5 à $600 \mu \mathrm{g} / \mathrm{kg}$, ce qui est inquiétant, vu son pouvoir cancérigène et les degrés élevés de contamination (Bullerman, 1981). 


\section{TABLEAU $3-T A B L E 3$}

Contamination des fromages par d'autres mycotoxines que l'aflatoxine $\mathbf{M}_{1}$ (seules les analyses positives pour une mycotoxine au moins sont rapportées)

\begin{tabular}{|c|c|c|c|c|c|c|c|c|c|c|}
\hline \multicolumn{2}{|l|}{ FROMAGES } & \multicolumn{8}{|c|}{ Nombre d'échantillons positifs } & \multirow[b]{2}{*}{ Références } \\
\hline Nature & $\begin{array}{l}\text { Nbre } \\
\text { d'échan- } \\
\text { tillons } \\
\text { analysés }\end{array}$ & $\begin{array}{l}\text { Citri- } \\
\text { nine }\end{array}$ & $\begin{array}{l}\text { Acide } \\
\text { cyclopia- } \\
\text { zonique }\end{array}$ & $\begin{array}{l}\text { Acide } \\
\text { myco- } \\
\text { phéno- } \\
\text { lique }\end{array}$ & $\begin{array}{l}\text { Ochra- } \\
\text { toxine }\end{array}$ & $\begin{array}{l}\text { Patu- } \\
\text { line }\end{array}$ & $\begin{array}{l}\text { Acide } \\
\text { pénicil- } \\
\text { lique }\end{array}$ & $\begin{array}{l}\text { Roque- } \\
\text { fortine }\end{array}$ & $\begin{array}{l}\text { Stérig- } \\
\text { mato- } \\
\text { cystine }\end{array}$ & \\
\hline Pâte dure & 48 & - & - & 4 & - & 1 & 3 & - & 3 & LAFONT et coll. (1979 c) \\
\hline $\begin{array}{l}\text { Pâte pressée } \\
\text { et croûte moisie }\end{array}$ & 39 & - & - & 7 & - & 4 & 5 & - & 0 & LAFONT et coll. (1979 c) \\
\hline Type camembert & 20 & - & 11 & - & - & - & - & - & - & LEBARS (1979 a) \\
\hline Pâte persillée & 110 & - & - & 41 & - & 0 & 1 & - & 0 & LAFONT et coll. (1979 c) \\
\hline Roquefort & 25 & - & - & 21 & - & - & - & - & - & LAFONT et coll. (1979 b) \\
\hline $\begin{array}{l}\text { Fromage fondu } \\
\text { (roquefort) }\end{array}$ & 2 & - & - & 2 & - & - & - & - & - & LAFONT et coll. (1979 b) \\
\hline $\begin{array}{l}\text { Fromage bleu } \\
\text { (allemand) }\end{array}$ & 12 & - & - & 3 & - & - & - & - & - & LAFONT et coll. (1979 b) \\
\hline Bleu des Causses & 6 & - & - & 3 & - & - & - & - & - & LAFONT et coll. (1979 b) \\
\hline Gorgonzola & 12 & - & - & 3 & - & - & - & - & - & LAFONT et coll. (1979 c) \\
\hline Fromages bleus & 12 & - & - & - & - & - & - & 12 & - & WARE et coll. (1980) \\
\hline $\begin{array}{l}\text { Sauce de fromages } \\
\text { bleus }\end{array}$ & 2 & - & - & - & - & - & - & 2 & - & WARE et coll. (1980) \\
\hline $\begin{array}{l}\text { Fromage sec } \\
\text { de chèvre }\end{array}$ & 18 & - & - & 0 & - & 1 & 2 & - & 0 & LAFONT et coll. (1979 c) \\
\hline Divers & 44 & 17 & - & - & 18 & - & - & - & - & JARVIS (1983) \\
\hline
\end{tabular}


Les travaux de Jarvis et ses coll. (Jarvis, 1983) ont permis, eux, de mettre en évidence l'ochratoxine (jusqu'à $260 \mu \mathrm{g} / \mathrm{kg}$ ) et la citrinine (pas plus de $50 \mu \mathrm{g} / \mathrm{kg}$ ) dans un certain nombre d'échantillons (tab. 3).

Le pénitrème $A$, mycotoxine responsable de tremblements et de spasmes musculaires, a été détecté comme contaminant naturel des fromages (Richard et Arp, 1979), mais il s'agissait d'une contamination par P.crustosum survenue dans un réfrigérateur ménager.

Enfin, il est important de signaler que l'aflatoxine $B_{1}$ peut être incidemment rencontrée dans les fromages du commerce (Bullerman, 1976 b).

\section{CONCLUSION}

Certes, il est du devoir des hygiénistes de se préoccuper de tous les risques sanitaires, aussi mineurs soient-ils, pour le consommateur. Mais il faut se garder d'être trop alarmiste. En effet, parmi toutes les mycotoxines pouvant se rencontrer dans les fromages, seule l'aflatoxine $M_{1}$ est à prendre sérieusement en considération, à notre avis, à cause de sa toxicité et de son pouvoir hépatocancérigène élevé, quoique plus faible que celui de l'aflatoxine $\mathrm{B}_{1}$. Les résultats des analyses effectuées par certains laboratoires sont à interpréter avec beaucoup de prudence, du fait de la nature des échantillons examinés, parfois choisis parmi les lots suspects.

Toutefois, il s'agit de rester vigilant car il suffit, par exemple, d'avoir un été humide aux Etats-Unis, comme en 1977, ou de laisser pénétrer en France des tourteaux d'arachides fortement contaminés, pour que le problème ressurgisse ici o!ı là. Un contrôle rigoureux de l'alimentation des femelles laitières reste donc la seule garantie pour une salubrité suffisante des fromages, en attendant que soient mises au point des méthodes de détoxification du lait qui soient efficaces, peu coûteuses et compatibles avec les contraintes technologiques.

La présence, sur les fromages, de micromycètes toxinogènes doit également être interprétée avec beaucoup de réserve. L'exemple le plus éclatant est certainement celui du P.roqueforti, capable de synthétiser la PR toxine sur des milieux de laboratoire mais pas sur les fromages (Guiraud et coll., 1977). Le P.caseicolum et le P.camemberti peuvent également être toxinogènes. Cependant, la température peut avoir un effet très marqué sur la toxinogénèse des souches, ce qui permet de suggérer que des souches peu toxinogènes aux températures d'affinage des fromages pourraient être sélectionnées pour un usage industriel (Le Bars, 1979 b).

Certes, les Aspergillus peuvent synthétiser des toxines cancérigènes telles que les aflatoxines ou la stérigmatocystine sur certains types de fromages, mais la contamination naturelle de ces produits par les micromycètes de ce genre reste assez rare, et leur toxinogénèse exige des températures relativement élevées. Le maintien des fro- 
mages aux basses températures permet donc de réduire considérablement les risques (Bullerman, 1977 b, 1981). D'ailleurs, même parmi les Penicillium, qui représentent la mycoflore dominante des fromages, ce sont les souches non toxinogènes qui tendent à prédominer aux températures de la réfrigération (Bullerman, 1981). En outre, les mycotoxines de Penicillium sont généralement beaucoup moins dangereuses que celles des Aspergillus, elles sont produites en faibles quantités, et sont relativement peu stables dans les fromages (Engel et Prokopek, 1979; Lieu et Bullerman, 1977 ; Stott et Bullerman, 1976).

Parmi les toxines de Penicillium, la citréoviridine peut être produite en quantité relativement élevée sur les fromages (Engel, 1978), mais elle est facilement reconnaissable à sa couleur jaune, et il est donc possible d'éliminer la partie contaminée. Bullerman (1981) discute cependant l'opportunité d'une telle pratique pour les moisissures en général, étant donné la diffusion possible des toxines à l'intérieur d'un fromage moisi superficiellement (Jarvis, 1983; Lie et Marth, 1967; Shih et Marth, 1972 a).

Mais les moyens de prévention demeurent certainement les plus indiqués en matière de mycotoxines comme dans d'autres domaines. Beaucoup de fromages, heureusement, présentent des moyens de défense efficaces contre la mycoflore de contamination tels que leurs micro-organismes propres ou leur croûte sèche et dure. Cependant, dans la majorité des cas, comme le souligne Bullerman (1977 b), un contrôle efficace des moisissures ne peut être assuré que par un programme associant le respect de bonnes règles hygiéniques à l'usine, l'usage de fongistatiques, le conditionnement sous-vide et, autant que possible, le stockage au froid.

\section{Bibliographie}

AfZal (M.), Cheems (R. A.), Chaudharay (R. A.) (1979). - Incidence of aflatoxins and aflatoxin producing fungi in animal feedstuffs. Mycopathologia, 69, 149-151.

Allcroft (R.), CARnAghan (R. B. A.) (1963). - Groundnut toxicity: an examination for toxin in human food products from animals fed toxic groundnut meal. Vet. Rec., 75, 259-263.

Applebaum (R. S.), Brackett (R. E.), Wiseman (D. W.), Marth (E. H.) (1982). Aflatoxin: toxicity to dairy cattle and occurrence in milk products. A review. J. Food Prot., 45, 752-777.

ARP (L. H.), RICHARD (J. L.) (1979). - Intoxication of dogs with the mycatoxin penitrem A. J. Am. Vet. Med. Assoc, 175, 565.

BLANC (B.), KARLESKIND (A.) (1981). - Données sur la contamination par l'aflatoxine $\mathrm{M}_{1}$ du lait et des produits laitiers en France. Résultats de 1046 analyses effectuées durant la période allant du $1^{\text {er }}$ septembre 1980 au 30 juin 1981. Le Lait, 61, 481-493.

Blanc (B.), Lauber (E.), Sieber (R.). 1983). - Fixation de l'aflatoxine sur les protéines du lait. Microbiologie, Aliments, Nutrition, 1, 163-177. 
Boutibonnes (J.), Jacouet (J.) (1967). - Recherches sur la production de toxine par Aspergillus flavus. Bull. Acad. Vét. Fr., 40, 393-403.

Brackett (R. E.), Applebaum (R. S.), Wiseman (D. W.), Marth (E. H.). (1982). Fate of aflatoxin $\mathbf{M}_{1}$ in Brick and Limburger-like cheese. J. Food Prot., 45, 553-556.

BracketT (R. E.), MARTH (E. H.) (1982 a). - Fate of aflatoxin $\mathbf{M}_{1}$ in Cheddar cheese and in process chesse spread. J. Food Prot., 45, 549-552.

Brackett (R. E.), Marth (E. H.) (1982 b). - Fate of aflatoxin $M_{1}$ in Parmesan and Mozzarella cheese. J. Food Prot., 45, 597-600.

Bullerman (L. B.) (1976 a). - Examination of Swiss cheese for incidence of mycotoxin producing molds. J. Food Sci., 41, 26-28.

Bullerman (L. B.) (1976 b). - Toxigenic potential of molds isolated from moldy cheese trimmings. Page 10 in Abstr. 63rd Annu. Mtg. Int. Assoc. Milk. Food Environ. Sanit. Inc, Arlington Heights, IL.

Bullerman (L. B.) (1977 a). - Incidence and control of mycotoxin producing molds in domestic and imported cheeses. Ann. Nutr. Alim., 31, 435-446.

Bullerman (L. B.) (1977 b). - Mold control in the cheese plant. Italian Cheese J., $6,1-8$.

Bullerman (L. B.) (1979). - Significance of mycotoxins to food safety and human health. J. Food Prot., 42, 65-86,

BUllerman (L. B.) (1980). - Incidence of mycotoxic molds in domestic and imported cheeses. J. Food Safety, 2, 47-58.

Bullerman (L. B.) (1981). - Public health significance of molds and mycotoxins in fermented dairy products. J .Dairy Sci., 64, 2439-2452.

Bullerman (L. B.), Olivigni (F.J.) (1974). - Mycotoxin producing potential of molds isolated from Cheddar cheese. J. Food Sci., 39, 1166-1168.

Corbion (B.), Fremy (J.M.) (1978), - Recherche des aflatoxines $B_{1}$ et $M_{1}$ dans les fromages de type "Camembert ». Le Lait, 58, 133-140.

Davis (N.), Diener (U. L.), Agnihotri (V. P.) (1967). - Production of aflatoxin B and $\mathrm{G}_{1}$ in a chemically defined medium. Mycopathol. Mycol. Appl., 31, 251-256.

El Gendy (S. M.), MaRTH (E. H.) (1981). - Growth and aflatoxin production by Aspergillus parasiticus in the presence of Lactobacillus casei. J. Food Prot., 44, 211-214.

ENGEL (G.) (1976). - Studies on the production of mycotoxins and their quantitative determination. 7. Synthesis of aflatoxins by Aspergillus flavus in the presence of sucrose or lactate as C-sources. Kiel. Miltchwirtsch. Forschungsber., 28,385 .

Engel (G.) (1978). - Bildung von Mykotoxinen auf Tilsiter Käse. Milchwissenschaft, 33, 201-203.

EnGel (G.) (1981). - Production of cyclopiazonic acid by Penicillinm camemberti and P. caseicolum. Jahresberichte 1981 der Bundesanstalt für Milchforschung in Kiel - B 33-B 34.

Engel (G.), Mulczewski (V.), ProkopeK (D.) Teuber (M.) (1982). - Strains-specific synthesis of mycophenolic acid by Penicillium roqueforti in blue veined cheese. Appl. Environn. Microbiol., 43, 1034-1040.

Engel (G.), PRoкорек (D.) (1979). - Kein Nachweis von Penicillium roqueforti toxin in Käse. Milchwissenschaft, 34, 272-274.

FranK (H. K.) (1968). - Diffusion of aflatoxins in food stuffs. J. Food Sci., 33, 98-100.

FranK (H. K.) (1974), - Aflatoxine. B. Behr's Verlag, Hambourg, 1974. 
Frank (H. K.), Orth (R.), Ivankovic (S.), Kuhlmann (M.), Schmahl (D.) (1977). Investigations on carcinogenic effects of Penicillium caseicolum and $P$. roqueforti in rats. Experientia, 33, 515-516.

Fremy (J.M.), Roiland (J.C.) (1979). - Devenir de l'aflatoxine $\mathrm{M}_{1}$ au cours de la fabrication du fromage de type "Camembert ». Ann. Nutr. Alim., 33, 619-630.

Gibel (W.), Wegner (K.), Wildner (G. P.) (1971). - Experimentelle Untersuchungen zur Frage einer kanzerogenen Wirkung von Penicillium camemberti var. candidum. Archiv. f. Geschwutstforsch., 38, 1-6.

Grant (D. W.) Carlson (F.W.) (1971). - Partitioning behavior of aflatoxin M in dairy products. Bull. Environm. Contam. Toxicol., 6, 521-524.

Gueguen (M.), Desfleurs (M.), Lemarinier (S.) (1978). - Penicillium roqueforti Thom, responsable d'un nouvel accident en fromagerie de pâtes molles. Le Lait, 58, 327-335.

Guiraud (I.), Piva (M. T.), Crouzet (J.), Galzy (P.) (1977). - Action de quelques facteurs du milieu sur l'excrétion de la PR toxine. Ann. Nutr. Alim., 31, 551-555.

Heathcote (J. G.), Hibbert (J. R.) (1978). - Aflatoxins: Chemical and biological aspects. Elsevier Scientific Publishing Co. New York, p. 4, 5, 7-9 et 44.

JacQuet (J.), BoutibonNes (P.) (1970). - Recherches sur les flavatoxines ou mieux flavacoumarines. Rev, Immuno., 34, 245-274.

JACQUet (J.), LAFONT (J.), LAFONT (P.) (1981). - Importance actuelle de la contamination des aliments par les mycotoxines. Mesures à prendre. Bull. Acad. Nat. Méd., 165, 359-364.

Jacouet (J.), TAnTAOUi-ElaRaKi (A.) (1975). - Recherche d'un milieu synthétique pour la croissance et la toxicogénèse des Aspergillus du groupe flavus. Bull. Acad. Vét. Fr., 48, 457-466.

Jacouet (J.), TANTAOUI-ElaRaki (A.) (1976). - Les produits laitiers comme milieux de culture et de toxicogènèse des Aspergillus du groupe flavus. Cas particulier des fromages. C.R. Acad. Agr., 62, 208-217.

Jacouet (J.), Teherani (A.) (1974). - Présence exceptionnelle de l'aflatoxine dans certains produits d'origine animale. Rôle possible du poivre. Bull. Acad. Vét. Fr., 47, 313-315.

JARVIS (B.) (1971), - Factors affecting the production of mycotoxins. J. Appl. Bacteriol., 34, 199-213.

JARVIS (B.) (1983). - Mould and mycotoxins in mouldy cheeses. Microbiologie, Aliments, Nutrition, 1, 187-191.

KieRMeier (F.) (1974). - The significance of aflatoxins in the dairy industry. Annual Bulletin International Dairy Federation, document 30.

KieRMeIeR (F.), BUChNeR (M.) (1977 a). - Zur Verteilung von aflatoxin $\mathrm{M}_{1}$ auf Molke und Bruch bei der Käseherstellung. Z. Lebensm. Unters. Forsch., $164,82$.

KieRmeier (F.), BuchNeR (M.) (1977 b). - Verhalten von aflatoxin M $_{1}$ während der Reifung und Lagerung von Käse. Z. Lebensm. Unters. Forsch., 164, 87.

Kiermeier (F.), Groll (D.) (1970). - Zur Aflatoxin B B $_{1}$-Bildung in Käsen. Z. Lebensm. Unters. Forsch., 143, 81-89.

KiERMeIER (F.), KraUS (P. V.) (1980). - The possible presence of sterigmatocystin in milk and its behavior in cheese. Z. Lebensm. Unters. Forsch., 170, 421-424.

KreRmeier (F.), Mashaley (R.) (1977). - Einfluss der molkereitechnischen Behandlung der Rohmilch auf des Aflatoxin $M_{1}$ Gejalt der daraus hergestellten Produkte. Z. Lebensm. Unters. Forsch., 164, 183-187. 
Kiermeier (F.), Weiss (G.) (1976). - Zur Untersuchung von Milch und Milchprodukten auf die Aflatoxnie $\mathrm{B}_{1}, \mathrm{~B}_{2}, \mathrm{G}_{1}, \mathrm{G}_{2}$ und $\mathrm{M}_{1}$. Z . Lebensm. Unters. Forsch, $160,337-344$.

KIERMEIER (F.), ZiereR (E.) (1975), - Effect of pimaricin on moulds and their aflatoxin formation in cheese. Z. Lebensm. Unters. Forsch., 157, 253-262.

Lafont (P.), Debeaupuis (J. P.), Gaillardin (M.), Payen (J.) (1979 a). - Production of mycophenolic acid by Penicillium roqueforti strains. Appl. Environ. Microbiol., 37, 365 .

Lafont (P.), Lafont (J.), Payen (J.), Chany (E.), Bertin (G.), Frayssinet (C.) (1976). - Toxin production by 50 strains of Penicillium used in the cheese industry. Fd. Cosmet. Toxicol, 14, 137-140.

Lafont (P.), Siriwardana (M. G.), Combemale (I.), Lafont (J.) (1979 b). - Mycophenolic acid in marketed cheeses. $F d$. Cosmet. Toxicol., 17, 147-149.

Lafont (P.), Siriwardana (M. B.), Lafont (J.) (1979 c). - Contamination des fromages par des métabolites fongiques. Méd. et Nutr., 15, 257.

Landers (D.E.), Davis (N.D.), Diener (U.L.) (1967). - Influence of atmospheric gases on aflatoxin production by Aspergillus flavus in peanuts. Phytopathol., 57, 1086-1090.

Lebars (J.) (1979 a). - Cyclopiazonic acid production by Penicillium camemberti and natural occurrence of this mycotoxin in cheese. Appl. Environ. Microbiol., $38,1052-1055$.

Lebars (J.) (1979 b). - Cyclopiazonic acid bioproduction by Penicillium camemberti. Effect of temperature on individual strains. Ann. Rech. Vét., 10, 601-602.

LIE (J. L.), MaRTH (E. H.) (1967). - Formation of aflatoxin in Cheddar cheese by Aspergillus flavus and Aspergillus parasiticus. J. Dairy Sci., 50, 1708-1710.

LIE (J. L.), MARTH (E. H.) (1968). - Formation of aflatoxin in Cheddar cheese by Aspergillus flavus and Aspergillus parasiticus in casein substrate at different $\mathrm{pH}$ values. J. Dairy Sci., 51, 1743-1747.

Lieu (F. Y.), Bullerman (L. B.) (1977). - Production and stability of aflatoxins, penicillic acid and patulin in several substrates. J. Food Sc., 42, 1222.

Masri (M. S.), Garcia (V. C.), Page (J.R.) (1969), - Aflatoxin M content of milk from cows fed know amounts of aflatoxin. Vet. Rec., 84, 146-147.

Mateles (R. I.), Adye (J. C.) (1965). - Production of aflatoxins in submerged cultures. Appl. Microbiol., 13, 208-211.

Mc. Kinney (J. D.), Cavanagh (G. C.) (1977). - Extraction of "bound» aflatoxin. Zeszyty Problemowe Postepow Nauk Rolniczych. 189,247-253.

Mc. Kinney (J. D.), Cavanagh (G. C.), Bell (J. T.), Hoverslund (A. S.), Nelson (D. M.), PeARson (J.), SelKirK (R. J.) (1973). - Effects of ammoniation of aflatoxins in rations fed lactating cows. J. Am. Oil. Chem. Soc., 50, 79-84.

Mirocha (C. J.), Pathre (S. V.), Robinson (T. S.) (1981). - Comparative metabolism of zearalenone and transmission into bovine milk. Fd. Cosmet. Toxicol., 19, 25-30.

Moreau (C.) (1976). - Les mycotoxines dans les produits laitiers. Le Lait, 56, 286-303.

Moreau (C.) (1980). - Penicilium roqueforti : morphologie, physiologie, importance en fromagerie, mycotoxines. Revue bibliographique, Le Lait, 60, 254-271.

Morris (H. A.), CASTBERg (H. B.) (1980). - Control of surface growth on blue cheese using pimaricin. Cultured Dairy Prod. J., May, 21-23.

Morris (H. A.), Hart (P. A.) (1978). - Pimaricin - Wat is it ? Cultured Dairy Prod. $J$., August, 22-24. 
Northolt (M. D.), van Egmond (H. P.), Paulsch (W. E.) (1978), - Patulin production by some fungal species in relation to water activity and temperature. J. Foot Prot., 41, 885.

Northolt (M. D.), van Egmond (H. P.), Paulsch (W. E.) (1979 a). - Penicillic acid production by some fungal species in relation to water activity and temperature. J. Food Prot., 42, 476.

Northolt (M. D.), van Egmond (H. P.), Paulsch (W. E.) (1979 b). - Ochratoxin A production by some fungal species in relation to water activity and temperature. J. Food Prot., 42, 485.

Northolt (M. D.), van Egmond (H. P.), Soentoro (P.), Dejill (E.) (1980). - Fungal growth and the presence of sterigmatocystin in hard cheese. J. Assoc. Offic. Anal. Chem., 63, 115.

Ohmono (S.), Sato (T.), Utagawa (T.), ABE (M.) (1975), - Isolation of festuclavine and three new indole alkaloids, roquefortine $\mathrm{A}, \mathrm{B}$ and $\mathrm{C}$ from cultures of Penicillium roqueforti. Agric. Biol. Chem., 39, 1133-1134.

Oldham (L. S.) , Oehme (F. W.), Kelley (D. C.) (1971). - Production of aflatoxin in prepackaged luncheon meat and cheese at refrigerator temperatures. J. Milk. and Food Technol., 34, 349-351.

Olivigni (F. J.), Bullerman (L. B.) (1977). - Simultaneous production of penicillic acid and patulin by a Penicillium species isolated from Cheddar cheese. J. Food Sci., 42, 1654-1657.

Polan (C. E.), Hayes (J. R.), Campbell (T. C.) (1974), - Consumption and fate of aflatoxin $\mathrm{B}_{1}$ by lactating cows. J. Agric. Food Chem., 22, 635-638.

Polmmeier (M. M.), Bullerman (L. B.) (1978). - Ochratoxin production by a Penicillium species isolated from cheese. Abst. 65th Annu. Mtg Inst. Assoc. Milk, Food and Environ. Sanit. Inc., Kansas City, MO.

Polzhofer (K.) (1977 a). - Aflatoxinbestimmung in Milch und Milchprodukten. Z. Lebensm. Unters. Forsch., 163, 175-177.

Polzhofer (K. P.) (1977 b). - Hitzestabilität von aflatoxin $\mathrm{M}_{1}$. Z. Lebensm. Unters. Forsch., 164, 80-81.

PRZYBYLSKi (K. S.), Bullerman (L. B.) (1980). - Influence of sorbic acid on viability and ATP content of conidia of Aspergillus parasiticus. J. Food Sci., 45, 375-385.

Purchase (I. F. H.), Steyn (M.), Rinsma (R.), Tustin (R. C.) (1972). - Reduction of the aflatoxin $\mathrm{M}$ content of milk by processing. Food Cosmet. Toxicol., 10, 383-387.

Ribelin (W. E.) (1978). - Ochratoxicosis in cattle. In «Mycotoxic Fungi, Mycotoxins, Mycotoxicoses", Ed. T.D. Willie and L.G. Morehouse, M. Dekker, Inc. New York and Basel, vol. 2, p. 28-36.

RICHARD (J. L.), ARP (L. H.) (1979). - Natural occurrence of the mycotoxin penitrem A in moldy cream cheese. Mycopathologia, 67, 107.

ScotT (P. M.), KENNEDy (B. P. C.) (1976). - Analysis of blue cheese for roquefortine and other alkaloids from Penicillium roqueforti. J. Agr. Food Chem., 24, 865.

SHAHANI (K. M.), Goldberg (H. S.) (1972). - Inhibitory effect of pimaricin upon mold growth, metabolism and toxin production. Intern. Sympos. Control of Mycotoxins (IUPAC) Kungälv (Göteborg), Sweden, p. 22.

SHIH (C. N.), MARTH (E. H.) (1972 a). - Experimental production of aflatoxin on Brick cheese. J. Milk Food Technol., 35, 585-587.

ShiH (C. N.), Marth (E.H.) (1972 b). - Production of aflatoxin in a medium fortified with sodium chloride. J. Dairy Sci., 55, 1415-1419.

SHIH (C. N.), MARTH (E. H.) (1973). - Aflatoxin produced by Aspergillus parasiticus when incubated in the presence of different gases. J. Milk Food Technol., 36, 421-425. 
Stoloff (L.), Trucksess (M.), Hardin (N.), Francis (O. J.), Hayes (J.R.), Polan (C. E.), Campbell (T. C.) (1975). - Stability of aflatoxin $\mathrm{M}_{1}$ in milk. J. Dairy Sci., 58, 1789-1793.

Stoloff (L.), Wood (G.), Carter (L. Jr) (1981). - Aflatoxin $M_{1}$ in manufactured dairy products produced in the United States in 1979. J. Dairy Sci., 64, 2426-2430.

Stott (W. T.), Bullerman (L. B.) (1975). - Influence of carbohydrate and nitrogen source on patulin production by Penicillium patulum. Appl. Microbiol., 30, 85.

StotT (W. T.), Bullerman (L. B.) (1976). - Instability of patulin in Cheddar cheese. J. Food Sci., 41, 201-203.

Stubblefield (R. D.), Shannon (G. M.) (1974). - Aflatoxin $\mathbf{M}_{1}$ : analysis in dairy products and distribution in dairy foods made from artificially contaminated milk. J. Assoc. Off. Anal. Chem., 57, 847-851.

Tantaoui-Elaraki (A.), Khabbazi (N.), Bardach (J.) (1983). - Les moisissures toxinogènes et les mycotoxines dans le lait et les produits laitiers. Maghreb Vétérinaire, 1, 27-40.

Teuber (M.), ENGel (G.) (1983). - Low risk of mycotoxin production in chesse. Microbiologie, Aliments, Nutrition, 1, 193-197.

Torrey (G. S.), Marth (E. H.) (1977). - Temperatures in home refrigerators and mold growth at refrigeration temperatures. J. Food Prot., 40, 393.

van Egmond (H. P.), Paulsch (W. E.), Veringa (H. A.), Schuller (P. L.) (1977. The effect of processing on the aflatoxin $\mathrm{M}_{1}$ content of milk and milk products. Arch. Inst. Pasteur (Tunis), 54, 381-390.

Ware (G. M.), Thorpe (C. W.), Pohland (A. E.) (1980). - Determination of roquefortine in blue cheese and blue cheese dressing by High Presure Liquid Chromatography with ultraviolet and electrochemical detectors. J. Assoc. Off. Anal. Chem., 63, 637-641.

WeckBaCh (L. S.), MaRth (E. H.) (1977). - Aflatoxin production by Aspergillus parasiticus in a competitive environment. Mycopathologia, 62, 39-45.

Wei (R. D.), Still (P. E.), Smalley (E. B.), Schnoes (H. K.), Strong (F. M.) (1973). Isolation and partial characterization of a mycotoxin from Penicillium roqueforti. Appl. Microbiol., 25, 111-114.

Wiseman (D. W.), MARTH (E. H.) (1981). - Growth and aflatoxin production by Aspergillus parasiticus when in the presence of Streptococcus lactis. Mycopathologia, 73, 49-56.

Yoshizawa (T.), Mirocha (C. J.), Behrens (J. C.), Swanson (S. P.) 1981). - Metabolic fate of T-2 toxin in a lactating cow. Food Cosmet. Toxicol., 19, 31-39.

Youssef (A. E.), MARTH (E. H.) (1981). - Growth and synthesis of aflatoxin by Aspergillus parasiticus in the presence of sorbic acid. J. Food Prot., 44, 736-741. 